DESY 08-150

ISSN 0418-9833

September 2008

\title{
New relationships between Feynman integrals
}

\author{
O. V. TARASOV* \\ II. Institut für Theoretische Physik, Universität Hamburg, \\ Luruper Chaussee 149, 22761 Hamburg, Germany
}

\begin{abstract}
New types of relationships between Feynman integrals are presented. It is shown that Feynman integrals satisfy functional equations connecting integrals with different values of scalar invariants and masses. A method is proposed for obtaining such relations. The derivation of functional equations for one-loop propagator- and vertex - type integrals is given. It is shown that a propagator - type integral can be written as a sum of two integrals with modified scalar invariants and one propagator massless. The vertex - type integral can be written as a sum over vertex integrals with all but one propagator massless and one external momentum squared equal to zero. It is demonstrated that the functional equations can be used for the analytic continuation of Feynman integrals to different kinematic domains.
\end{abstract}

PACS numbers: 02.30.Gp, 02.30.Ks, 12.20.Ds, 12.38.Bx

Keywords: Feynman integrals, functional equations, Appell hypergeometric function

*On leave of absence from Joint Institute for Nuclear Research, 141980 Dubna (Moscow Region), Russia. 


\section{A method for deriving functional equations}

Feynman integrals play an important role in making precise perturbative predictions in quantum field theory. As is well-known, these integrals satisfy recurrence relations [1]- 4]. In general such relations connect several integrals $I_{1, n}, \ldots, I_{N, n}$ with $n$ internal lines and integrals with a lesser number of internal lines. They may be written in the following form

$$
\sum_{i=1}^{N} Q_{i}\left(\left\{m_{j}\right\},\left\{s_{q}\right\}, \nu_{l}, d\right) I_{i, n}=\sum_{\substack{r<n \\ k}} R_{k, r}\left(\left\{m_{j}\right\},\left\{s_{m}\right\}, \nu_{l}, d\right) I_{k, r},
$$

where $I_{k, r}$ stands for integrals with $r$ internal lines, arbitrary powers of propagators $\nu_{j}$ and arbitrary shifts of the space-time dimension $d ;\left\{s_{q}\right\}$ is a set of independent scalar invariants that may be formed from the external momenta. $Q_{i}, R_{k}$ are ratios of polynomials depending on $\left\{s_{r}\right\}$, masses $m_{j}, \nu_{l}$ and $d$. On the left-hand side of (1.1) we combined integrals with $n$ internal lines and on the right hand side integrals with a lesser number of lines.

The key idea in the derivation of the functional equations is to remove integrals with the maximal number of lines from the relations (1.1) by an appropriate choice of scalar invariants $\left\{s_{q}\right\}$, masses $m_{j}^{2}$, powers of propagators $\nu_{j}$ and space - time dimension $d$.

In order to obtain functional equations from a given equation (1.1) one should first solve the polynomial system of equations

$$
Q_{i}\left(\left\{m_{j}\right\},\left\{s_{q}\right\}, \nu_{l}, d\right)=0, \quad i=1, \ldots, N
$$

with respect to $\left\{s_{q}\right\},\left\{m_{i}\right\}, \nu_{l}, d$ and then take those solutions for which not all coefficients in front of integrals on the right-hand side of Eq.(1.1) are vanishing. In many cases functional equations can be obtained from (1.1) by choosing only kinematic variables $\left\{s_{q}\right\}$ and masses $\left\{m_{i}\right\}$.

We illustrate the method by considering the derivation of the functional equations for a one-loop integral depending on $n-1$ independent external momenta:

$$
I_{n}^{(d)}\left(\left\{m_{l}^{2}\right\} ;\left\{p_{i r}\right\}\right)=\int \frac{d^{d} q}{i \pi^{d / 2}} \prod_{j=1}^{n} \frac{1}{\left[\left(q-p_{j}\right)^{2}-m_{j}^{2}\right]^{\nu_{j}}},
$$

where

$$
p_{i r}=\left(p_{i}-p_{r}\right)^{2} .
$$

Here and below, the usual causal prescription for the propagators is understood, i.e. $1 /\left[q^{2}-\right.$ $\left.m^{2}\right] \leftrightarrow 1 /\left[q^{2}-m^{2}+i 0\right]$. Any relation of the form (1.1) can be used for deriving functional equations. In this paper we will use the following relation [4], [5]:

$$
\begin{aligned}
G_{n-1} \nu_{j} \mathbf{j}^{+} I_{n}^{(d+2)}\left(\left\{m_{l}^{2}\right\} ;\left\{p_{i r}\right\}\right) & -\left(\partial_{j} \Delta_{n}\right) I_{n}^{(d)}\left(\left\{m_{l}^{2}\right\} ;\left\{p_{i r}\right\}\right) \\
= & \sum_{k=1}^{n}\left(\partial_{j} \partial_{k} \Delta_{n}\right) \mathbf{k}^{-} I_{n}^{(d)}\left(\left\{m_{l}^{2}\right\} ;\left\{p_{i r}\right\}\right)
\end{aligned}
$$

where the operators $\mathbf{j}^{ \pm}$etc. shift the indices $\nu_{j} \rightarrow \nu_{j} \pm 1, G_{n-1}$ is the Gram determinant

$$
G_{n-1}=-2^{n}\left|\begin{array}{cccc}
\left(p_{1}-p_{n}\right)\left(p_{1}-p_{n}\right) & \left(p_{1}-p_{n}\right)\left(p_{2}-p_{n}\right) & \ldots & \left(p_{1}-p_{n}\right)\left(p_{n-1}-p_{n}\right) \\
\left(p_{1}-p_{n}\right)\left(p_{2}-p_{n}\right) & \left(p_{2}-p_{n}\right)\left(p_{2}-p_{n}\right) & \ldots & \left(p_{2}-p_{n}\right)\left(p_{n-1}-p_{n}\right) \\
\vdots & \vdots & \ddots & \vdots \\
\left(p_{1}-p_{n}\right)\left(p_{n-1}-p_{n}\right) & \left(p_{2}-p_{n}\right)\left(p_{n-1}-p_{n}\right) & \ldots & \left(p_{n-1}-p_{n}\right)\left(p_{n-1}-p_{n}\right)
\end{array}\right|
$$


and $\Delta_{n}$ is the modified Cayley determinant defined as:

$$
\Delta_{n}=\mid \begin{array}{cccc}
2 m_{1}^{2} & m_{1}^{2}+m_{2}^{2}-p_{12} & \ldots & m_{1}^{2}+m_{n}^{2}-p_{1 n} \\
m_{1}^{2}+m_{2}^{2}-p_{12} & 2 m_{2}^{2} & \cdots & m_{2}^{2}+m_{n}^{2}-p_{2 n} \\
\vdots & \vdots & \ddots & \vdots \\
m_{1}^{2}+m_{n}^{2}-p_{1 n} & m_{2}^{2}+m_{n}^{2}-p_{2 n} & \ldots & 2 m_{n}^{2} \\
\partial_{j} \equiv \frac{\partial}{\partial m_{j}^{2}} .
\end{array}
$$

We assume that the external momenta are not restricted to some specific integer dimension and therefore $G_{n-1}$ and $\Delta_{n}$ do not satisfy any condition specific to a particular value of the space-time dimension.

In the present paper we will consider functional equations only for integrals $I_{n}^{(d)}$ with the first powers of propagators. Setting all $\nu_{k}=1 \mathrm{in} \mathrm{Eq.} \mathrm{(1.5)} \mathrm{yields} \mathrm{an} \mathrm{equation} \mathrm{of} \mathrm{the} \mathrm{form} \mathrm{(1.1)}$ connecting integrals with $n$ and $n-1$ lines. Functional equations for the integral $I_{n-1}^{(d)}$ can be obtained for each particular $j$ by imposing two conditions:

$$
G_{n-1}=0, \quad \partial_{j} \Delta_{n}=0,
$$

and solving them by an appropriate choice of scalar invariants $p_{i j}$ and masses. There are only $n-1$ independent systems of relations of the type (1.8), because

$$
\sum_{k=1}^{n} \partial_{k} \Delta_{n}=-G_{n-1} .
$$

Since $G_{n-1}$ and $\partial_{j} \Delta_{n}$ are nonlinear in $p_{i j}$ and masses, each system of equations may have several solutions. The number of functional equations is less than the number of possible solutions. This is firstly because coefficients in front of integrals on both sides of Eq. (1.1) are simultaneously zero for some solutions, and secondly, because not all functional equations are independent.

\section{Functional equations for the one-loop propagator - type integral}

In accordance with our method described in the previous section, functional equations for the integral $I_{2}^{(d)}$ can be obtained from equation (1.5) taken at $n=3, \nu_{1}=\nu_{2}=\nu_{3}=1$. We will not derive all possible functional equations, restricting ourselves only to the case $j=1$ in (1.5):

$$
\begin{aligned}
G_{2} \mathbf{1}^{+} I_{3}^{(d+2)}\left(m_{1}^{2}, m_{2}^{2}, m_{3}^{2} ; p_{23}, p_{13}, p_{12}\right) & -\left(\partial_{1} \Delta_{3}\right) I_{3}^{(d)}\left(m_{1}^{2}, m_{2}^{2}, m_{3}^{2} ; p_{23}, p_{13}, p_{12}\right) \\
& =2\left(p_{12}+p_{23}-p_{13}\right) I_{2}^{(d)}\left(m_{1}^{2}, m_{2}^{2} ; p_{12}\right) \\
& +2\left(p_{13}+p_{23}-p_{12}\right) I_{2}^{(d)}\left(m_{1}^{2}, m_{3}^{2} ; p_{13}\right) \\
& -4 p_{23} I_{2}^{(d)}\left(m_{2}^{2}, m_{3}^{2} ; p_{23}\right)
\end{aligned}
$$

where

$$
\begin{aligned}
& I_{3}^{(d)}\left(m_{j}^{2}, m_{k}^{2}, m_{l}^{2} ; p_{k l}, p_{j l}, p_{j k}\right)=\int \frac{d^{d} q}{i \pi^{d / 2}} \frac{1}{\left[\left(q-p_{j}\right)^{2}-m_{j}^{2}\right]\left[\left(q-p_{k}\right)^{2}-m_{k}^{2}\right]\left[\left(q-p_{l}\right)^{2}-m_{l}^{2}\right]} \\
& I_{2}^{(d)}\left(m_{j}^{2}, m_{k}^{2} ; p_{j k}\right)=\int \frac{d^{d} q}{i \pi^{d / 2}} \frac{1}{\left[\left(q-p_{j}\right)^{2}-m_{j}^{2}\right]\left[\left(q-p_{k}\right)^{2}-m_{k}^{2}\right]}
\end{aligned}
$$


In order to remove integrals $I_{3}^{(d)}, I_{3}^{(d+2)}$ from this relation two conditions must be fulfilled:

$$
\begin{aligned}
G_{2}= & 2 p_{12}^{2}+2 p_{13}^{2}+2 p_{23}^{2}-4 p_{12} p_{13}-4 p_{12} p_{23}-4 p_{13} p_{23}=0 \\
\partial_{1} \Delta_{3}= & 2 p_{23}\left(p_{13}+p_{12}-p_{23}\right)-4 m_{1}^{2} p_{23} \\
& \quad+2 m_{2}^{2}\left(p_{23}+p_{13}-p_{12}\right)+2 m_{3}^{2}\left(p_{23}+p_{12}-p_{13}\right)=0 .
\end{aligned}
$$

One can solve this system of equations with respect to $p_{13}$ and $p_{23}$. The nontrivial solutions of the system (2.12) are:

$$
\begin{aligned}
& p_{13}=s_{13}\left(m_{1}^{2}, m_{2}^{2}, m_{3}^{2}, p_{12}\right)=\frac{\Delta_{12}+2 p_{12}\left(m_{1}^{2}+m_{3}^{2}\right)-\left(p_{12}+m_{1}^{2}-m_{2}^{2}\right) \lambda}{2 p_{12}}, \\
& p_{23}=s_{23}\left(m_{1}^{2}, m_{2}^{2}, m_{3}^{2}, p_{12}\right)=\frac{\Delta_{12}+2 p_{12}\left(m_{2}^{2}+m_{3}^{2}\right)+\left(p_{12}-m_{1}^{2}+m_{2}^{2}\right) \lambda}{2 p_{12}}
\end{aligned}
$$

where

$$
\begin{gathered}
\lambda= \pm \sigma\left(p_{12}-m_{1}^{2}+m_{2}^{2}\right) \sqrt{\Delta_{12}+4 p_{12} m_{3}^{2}}, \\
\sigma(x)=\left\{\begin{array}{lll}
+1 & \text { if } \quad x \geq 0, \\
-1 & \text { if } \quad x<0,
\end{array}\right. \\
\Delta_{i j}=p_{i j}^{2}+m_{i}^{4}+m_{j}^{4}-2 p_{i j} m_{i}^{2}-2 p_{i j} m_{j}^{2}-2 m_{i}^{2} m_{j}^{2} .
\end{gathered}
$$

Substituting Eq. (2.13) into Eq. (2.10) yields the following relation:

$$
\begin{aligned}
I_{2}^{(d)}\left(m_{1}^{2}, m_{2}^{2} ; p_{12}\right) & =\frac{p_{12}+m_{1}^{2}-m_{2}^{2}-\lambda}{2 p_{12}} I_{2}^{(d)}\left(m_{1}^{2}, m_{3}^{2} ; s_{13}\left(m_{1}^{2}, m_{2}^{2}, m_{3}^{2}, p_{12}\right)\right) \\
& +\frac{p_{12}-m_{1}^{2}+m_{2}^{2}+\lambda}{2 p_{12}} I_{2}^{(d)}\left(m_{2}^{2}, m_{3}^{2} ; s_{23}\left(m_{1}^{2}, m_{2}^{2}, m_{3}^{2}, p_{12}\right)\right) .
\end{aligned}
$$

All arguments of the integral $I_{2}^{(d)}$ on the left - hand side of (2.17) are arbitrary. At the same time, the last argument in integrals on the right - hand side satisfy conditions (2.12). The mass $m_{3}$ in the equation is an arbitrary parameter and can be chosen at will. Setting $m_{3}=0$ in Eqs. (2.13)-(2.17), yields

$$
\begin{aligned}
I_{2}^{(d)}\left(m_{1}^{2}, m_{2}^{2} ; p_{12}\right) & =\frac{p_{12}+m_{1}^{2}-m_{2}^{2}-\alpha_{12}}{2 p_{12}} I_{2}^{(d)}\left(m_{1}^{2}, 0 ; s_{13}\right) \\
& +\frac{p_{12}-m_{1}^{2}+m_{2}^{2}+\alpha_{12}}{2 p_{12}} I_{2}^{(d)}\left(0, m_{2}^{2} ; s_{23}\right)
\end{aligned}
$$

where

$$
\begin{gathered}
s_{13}=\frac{\Delta_{12}+2 p_{12} m_{1}^{2}-\left(p_{12}+m_{1}^{2}-m_{2}^{2}\right) \alpha_{12}}{2 p_{12}}, \\
s_{23}=\frac{\Delta_{12}+2 p_{12} m_{2}^{2}+\left(p_{12}-m_{1}^{2}+m_{2}^{2}\right) \alpha_{12}}{2 p_{12}}, \\
\alpha_{12}= \pm \sigma\left(p_{12}-m_{1}^{2}+m_{2}^{2}\right) \sqrt{\Delta_{12}} .
\end{gathered}
$$

The analytic expression for the integral $I_{2}^{(d)}\left(0, m^{2} ; p^{2}\right)$ is [6], [7]:

$$
I_{2}^{(d)}\left(0, m^{2} ; p^{2}\right)=I_{2}^{(d)}\left(0, m^{2} ; 0\right){ }_{2} F_{1}\left[\begin{array}{c}
1,2-\frac{d}{2} ; \frac{p^{2}}{m^{2}} \\
\frac{d}{2} ;
\end{array}\right.
$$


where

$$
I_{2}^{(d)}\left(0, m^{2} ; 0\right)=-\Gamma\left(1-\frac{d}{2}\right) m^{d-4}
$$

Thus, relations (2.18) and (2.21) give us the analytic result for the integral $I_{2}^{(d)}$ with arbitrary masses and external momentum squared. Our result is in agreement with that presented in [7].

Setting $m_{2}^{2}=0$ in equation (2.18), assuming $\left|p_{12}\right|>m_{1}^{2}$ and taking solution (2.19) corresponding to the + sign in formula (2.20) yields

$$
I_{2}^{(d)}\left(m_{1}^{2}, 0 ; p_{12}\right)=\frac{m_{1}^{2}}{p_{12}} I_{2}^{(d)}\left(m_{1}^{2}, 0 ; \frac{m_{1}^{4}}{p_{12}}\right)+\frac{\left(p_{12}-m_{1}^{2}\right)}{p_{12}} I_{2}^{(d)}\left(0,0 ; \frac{\left(p_{12}-m_{1}^{2}\right)^{2}}{p_{12}}\right) .
$$

The first term on the right - hand side is the same integral $I_{2}^{(d)}$ as on the left - hand side, but with the last argument inverted. The second term corresponds to the simple integral $I_{2}^{(d)}$ with both propagators massless:

$$
I_{2}^{(d)}\left(0,0 ; p^{2}\right)=\frac{1}{i \pi^{d / 2}} \int \frac{d^{d} k_{1}}{k_{1}^{2}\left(k_{1}-p\right)^{2}}=\frac{-\pi^{\frac{3}{2}}\left(-p^{2}\right)^{\frac{d}{2}-2}}{2^{d-3} \Gamma\left(\frac{d-1}{2}\right) \sin \frac{\pi d}{2}} .
$$

Formula (2.23) can be applied to the analytic continuation of the integral $I_{2}^{(d)}\left(m_{1}^{2}, 0 ; p_{12}\right)$ into the region of large momenta $\left|p_{12}\right|>m_{1}^{2}$. It can also be used for the analytic continuation of the integrals $I_{2}^{(d)}$ on the right - hand side of (2.18). Therefore, the relations (2.18) and (2.23) describe the integral $I_{2}^{(d)}$ with arbitrary masses and momenta in the whole kinematic region.

It is interesting to note that equation (2.23) corresponds to the well-known formula for the analytic continuation of Gauss's hypergeometric function (2.21) (see, for example, Ref. [8]) :

$$
{ }_{2} F_{1}\left[\begin{array}{c}
1,2-\frac{d}{2} ; z \\
\frac{d}{2} ;
\end{array}\right]=\frac{1}{z}{ }_{2} F_{1}\left[\begin{array}{c}
1,2-\frac{d}{2} ; \frac{1}{z} \\
\frac{d}{2} ;
\end{array}\right]+\frac{\Gamma\left(\frac{d}{2}\right) \Gamma\left(\frac{d}{2}-1\right)}{\Gamma(d-2)}(-z)^{\frac{d}{2}-2}\left(1-\frac{1}{z}\right)^{d-3} .
$$

Indeed, substituting the explicit expressions (2.21), (2.24) into (2.23) and canceling common factors we obtain relation (2.25) with $z=p_{12} / m_{1}^{2}$.

\section{Functional equations for the one-loop vertex - type in- tegral}

Functional equations for the vertex - type integral $I_{3}^{(d)}$ will be derived in the same fashion as for the propagator - type integral. Setting $n=4, \nu_{1}=\ldots=\nu_{4}=1$ and $j=1$ in Eq.(1.5) yields:

$$
\begin{aligned}
& G_{3} \mathbf{1}^{+} I_{4}^{(d+2)}\left(\left\{m_{l}^{2}\right\} ;\left\{p_{i j}\right\}\right)-\left(\partial_{1} \Delta_{4}\right) I_{4}^{(d)}\left(\left\{m_{l}^{2}\right\} ;\left\{p_{i j}\right\}\right)= \\
& \quad\left(\partial_{1}^{2} \Delta_{4}\right) I_{3}^{(d)}\left(m_{2}^{2}, m_{3}^{2}, m_{4}^{2} ; p_{34}, p_{24}, p_{23}\right) \\
+ & \left(\partial_{1} \partial_{2} \Delta_{4}\right) I_{3}^{(d)}\left(m_{1}^{2}, m_{3}^{2}, m_{4}^{2} ; p_{34}, p_{14}, p_{13}\right) \\
+ & \left(\partial_{1} \partial_{3} \Delta_{4}\right) I_{3}^{(d)}\left(m_{1}^{2}, m_{2}^{2}, m_{4}^{2} ; p_{24}, p_{14}, p_{12}\right) \\
+ & \left(\partial_{1} \partial_{4} \Delta_{4}\right) I_{3}^{(d)}\left(m_{1}^{2}, m_{2}^{2}, m_{3}^{2} ; p_{23}, p_{13}, p_{12}\right) .
\end{aligned}
$$


One can obtain a functional equation for $I_{3}\left(m_{1}^{2}, m_{2}^{2}, m_{3}^{2} ; p_{23}, p_{13}, p_{12}\right)$ with arbitrary arguments by appropriately choosing the four variables: $p_{14}, p_{24}, p_{34}, m_{4}^{2}$. To remove the integrals $I_{4}^{(d)}, I_{4}^{(d+2)}$ from (3.26), two conditions should be satisfied

$$
G_{3}=0, \quad \partial_{1} \Delta_{4}=0 .
$$

This system of equations depends on 10 variables $p_{12}, p_{13}, p_{14}, p_{23}, p_{24}, p_{34}, m_{1}^{2}, m_{2}^{2}, m_{3}^{2}, m_{4}^{2}$ and it can be solved by excluding, for example, $p_{14}$ and $p_{34}$. There are four solutions of the system (3.27) but appropriate expressions are rather long and for this reason they will not be presented here. Instead we consider simplified situation, namely we set in Eq. (3.26) from the very beginning $m_{4}^{2}=0$, and impose the following conditions

$$
G_{3}=0, \quad \partial_{1} \Delta_{4}=0, \quad \partial_{1} \partial_{2} \Delta_{4}=0 .
$$

This system can be solved by an appropriate choice of $p_{14}, p_{34}, p_{24}$. There are several solutions of (3.28), but only for two of them are coefficients in front of integrals on the right hand side of (3.26) different from zero. These solutions are

$$
\begin{aligned}
p_{14} & =s_{14}^{(13)}, \\
p_{34} & =s_{34}^{(13)}, \\
p_{24} & =s_{24}\left(m_{1}^{2}, m_{3}^{2}, p_{23}, p_{13}, p_{12}\right) \\
& =\frac{\left(p_{12}+p_{23}-m_{1}^{2}-m_{3}^{2}\right) p_{13}+\left(p_{12}-p_{23}-m_{1}^{2}+m_{3}^{2}\right)\left(m_{3}^{2}-m_{1}^{2}+\alpha_{13}\right)}{2 p_{13}},
\end{aligned}
$$

where

$$
\begin{aligned}
& s_{14}^{(i j)}=\frac{\Delta_{i j}+2 m_{i}^{2} p_{i j}-\left(p_{i j}+m_{i}^{2}-m_{j}^{2}\right) \alpha_{i j}}{2 p_{i j}}, \\
& s_{34}^{(i j)}=\frac{\Delta_{i j}+2 m_{j}^{2} p_{i j}+\left(p_{i j}+m_{j}^{2}-m_{i}^{2}\right) \alpha_{i j}}{2 p_{i j}}, \\
& \alpha_{i j}= \pm \sigma\left(p_{i j}-m_{i}^{2}+m_{j}^{2}\right) \sqrt{\Delta_{i j}} .
\end{aligned}
$$

Substituting (3.29) into (3.26) leads to the following functional equation:

$$
\begin{aligned}
& I_{3}^{(d)}\left(m_{1}^{2}, m_{2}^{2}, m_{3}^{2} ; p_{23}, p_{13}, p_{12}\right)= \\
& \quad \frac{p_{13}+m_{3}^{2}-m_{1}^{2}+\alpha_{13}}{2 p_{13}} I_{3}^{(d)}\left(m_{2}^{2}, m_{3}^{2}, 0 ; s_{34}^{(13)}, s_{24}\left(m_{1}^{2}, m_{3}^{2}, p_{23}, p_{13}, p_{12}\right), p_{23}\right) \\
& +\frac{p_{13}-m_{3}^{2}+m_{1}^{2}-\alpha_{13}}{2 p_{13}} I_{3}^{(d)}\left(m_{1}^{2}, m_{2}^{2}, 0 ; s_{24}\left(m_{1}^{2}, m_{3}^{2}, p_{23}, p_{13}, p_{12}\right), s_{14}^{(13)}, p_{12}\right) .
\end{aligned}
$$

Relation (3.31) means that the integral $I_{3}^{(d)}$ with arbitrary arguments can always be expressed in terms of integrals with at least one massless propagator. The only exceptional case, when $p_{12}=p_{13}=p_{23}=0$, is trivial. In turn, integrals $I_{3}^{(d)}$ with one massless propagator can be represented as a sum over integrals with two massless propagators. Indeed, setting $m_{2}^{2}=0$ in Eq. (3.31) yields:

$$
\begin{aligned}
& I_{3}^{(d)}\left(m_{1}^{2}, 0, m_{3}^{2} ; p_{23}, p_{13}, p_{12}\right)= \\
& \quad \frac{p_{13}-m_{1}^{2}+m_{3}^{2}+\alpha_{13}}{2 p_{13}} I_{3}^{(d)}\left(0, m_{3}^{2}, 0 ; s_{34}^{(13)}, s_{24}\left(m_{1}^{2}, m_{3}^{2}, p_{23}, p_{13}, p_{12}\right), p_{23}\right) \\
& \quad+\frac{p_{13}+m_{1}^{2}-m_{3}^{2}-\alpha_{13}}{2 p_{13}} I_{3}^{(d)}\left(m_{1}^{2}, 0,0 ; s_{24}\left(m_{1}^{2}, m_{3}^{2}, p_{23}, p_{13}, p_{12}\right), s_{14}^{(13)}, p_{12}\right) .
\end{aligned}
$$


Taking into account the symmetry of the integral $I_{3}^{(d)}$ with respect to its arguments one can use Eq. (3.32) to express integrals on the right hand side of (3.31) in terms of integrals with two propagators massless. Thus in case when external momenta squared are different from zero the following relation holds:

$$
\begin{aligned}
& I_{3}^{(d)}\left(m_{1}^{2}, m_{2}^{2}, m_{3}^{2} ; p_{23}, p_{13}, p_{12}\right)= \\
& \frac{\left(p_{13}+m_{3}^{2}-m_{1}^{2}+\alpha_{13}\right)\left(p_{23}+m_{3}^{2}-m_{2}^{2}+\alpha_{23}\right)}{4 p_{13} p_{23}} \\
& \times I_{3}^{(d)}\left(m_{3}^{2}, 0,0 ; s_{24}\left(m_{2}^{2}, m_{3}^{2}, s_{34}^{(13)}, p_{23}, s_{24}\left(m_{1}^{2}, m_{3}^{2}, p_{23}, p_{13}, p_{12}\right)\right), s_{34}^{(23)}, s_{34}^{(13)}\right) \\
& +\frac{\left(p_{13}+m_{3}^{2}-m_{1}^{2}+\alpha_{13}\right)\left(p_{23}-m_{3}^{2}+m_{2}^{2}-\alpha_{23}\right)}{4 p_{13} p_{23}} \\
& \times I_{3}^{(d)}\left(m_{2}^{2}, 0,0 ; s_{24}\left(m_{2}^{2}, m_{3}^{2}, s_{34}^{(13)}, p_{23}, s_{24}\left(m_{1}^{2}, m_{3}^{2}, p_{23}, p_{13}, p_{12}\right)\right), s_{14}^{(23)}, s_{24}\left(m_{1}^{2}, m_{3}^{2}, p_{23}, p_{13}, p_{12}\right)\right) \\
& +\frac{\left(p_{13}-m_{3}^{2}+m_{1}^{2}-\alpha_{13}\right)\left(p_{12}+m_{2}^{2}-m_{1}^{2}+\alpha_{12}\right)}{4 p_{13} p_{12}} \\
& \times I_{3}^{(d)}\left(m_{2}^{2}, 0,0 ; s_{24}\left(m_{1}^{2}, m_{2}^{2}, s_{24}\left(m_{1}^{2}, m_{3}^{2}, p_{23}, p_{13}, p_{12}\right), p_{12}, s_{14}^{(13)}\right), s_{34}^{(12)}, s_{24}\left(m_{1}^{2}, m_{3}^{2}, p_{23}, p_{13}, p_{12}\right)\right) \\
& +\frac{\left(p_{13}-m_{3}^{2}+m_{1}^{2}-\alpha_{13}\right)\left(p_{12}-m_{2}^{2}+m_{1}^{2}-\alpha_{12}\right)}{4 p_{12} p_{13}} \\
& \times I_{3}^{(d)}\left(m_{1}^{2}, 0,0 ; s_{24}\left(m_{1}^{2}, m_{2}^{2}, s_{24}\left(m_{1}^{2}, m_{3}^{2}, p_{23}, p_{13}, p_{12}\right), p_{12}, s_{14}^{(13)}\right), s_{14}^{(12)}, s_{14}^{(13)}\right) .
\end{aligned}
$$

There is one further simplification of note. Setting $m_{1}^{2}=m_{3}^{2}=p_{24}=0$ in Eq. (3.26) from the very beginning and solving system of equations

$$
G_{3}=0, \quad \partial_{1} \Delta_{4}=0,
$$

with respect to $p_{14}$ and $p_{34}$ yields a nontrivial relationship:

$$
\begin{aligned}
& I_{3}^{(d)}\left(0, m^{2}, 0 ; p_{23}, p_{13}, p_{12}\right)= \\
& -\frac{\left[b\left(p_{23}, p_{12}\right)-\left(p_{12}+m^{2}\right) \alpha_{123}\right] m^{2}}{2 \Lambda_{3}} I_{3}^{(d)}\left(m^{2}, 0,0 ; \kappa_{14}, 0, p_{12}\right) \\
& -\frac{\left[b\left(p_{12}, p_{23}\right)+\left(p_{23}+m^{2}\right) \alpha_{123}\right] m^{2}}{2 \Lambda_{3}} I_{3}^{(d)}\left(m^{2}, 0,0 ; \kappa_{34}, 0, p_{23}\right) \\
& +\frac{\Lambda_{3}-p_{13}\left(p_{12} p_{23}-m^{4}\right)-m^{2}\left(p_{12}-p_{23}\right) \alpha_{123}}{2 \Lambda_{3}} I_{3}^{(d)}\left(0,0,0 ; \kappa_{34}, \kappa_{14}, p_{13}\right),
\end{aligned}
$$

where

$$
\begin{aligned}
& \kappa_{14}=\frac{a\left(p_{23}, p_{12}\right)+m^{2} b\left(p_{23}, p_{12}\right) \alpha_{123}}{2 \Lambda_{3}}, \\
& \kappa_{34}=\frac{a\left(p_{12}, p_{23}\right)-m^{2} b\left(p_{12}, p_{23}\right) \alpha_{123}}{2 \Lambda_{3}},
\end{aligned}
$$




$$
\begin{aligned}
& \Lambda_{3}=\left(m^{4}-p_{12} p_{23}\right)\left(p_{23}-p_{12}\right)-\left(p_{12}+m^{2}\right) b\left(p_{12}, p_{23}\right), \\
& a\left(p_{12}, p_{23}\right)=m^{2}\left(p_{12}-p_{23}\right)^{2}\left(p_{23}-m^{2}\right)+\left[2\left(m^{2}-p_{23}\right) p_{12}-m^{2} p_{13}\right]\left(p_{23}+m^{2}\right) p_{13}, \\
& b\left(p_{12}, p_{23}\right)=\left(m^{2}+p_{13}+p_{12}-p_{23}\right) p_{23}+m^{2}\left(p_{13}-p_{12}\right), \\
& \alpha_{123}=\sigma\left(b\left(p_{12}, p_{23}\right)\right) \sqrt{\Delta_{123}}, \\
& \Delta_{123}=p_{12}^{2}+p_{13}^{2}+p_{23}^{2}-2 p_{12} p_{13}-2 p_{12} p_{23}-2 p_{13} p_{23} .
\end{aligned}
$$

Therefore, by using Eq.(3.35) we can represent the integral $I_{3}^{(d)}$ with arbitrary masses and nonzero kinematic variables as a combination of integrals with two massless propagators, one momentum squared equal to zero and integrals with all propagators massless. An analytic result for the integral $I_{3}^{(d)}$ with all propagators massless is known in terms of ${ }_{2} F_{1}$ functions (see Ref. [9]).

Integrals $I_{3}^{(d)}$ with two massless propagators on the right - hand side of (3.35) can be evaluated analytically. In the kinematic region $\left|p_{12}\right| \leq m^{2}$ and $\left|p_{13}\right| \leq m^{2}$ we find

$$
\begin{gathered}
I_{3}^{(d)}\left(0, m^{2}, 0 ; 0, p_{13}, p_{12}\right)=-\frac{I_{2}^{(d)}\left(0,0 ; p_{13}\right)}{m^{2}}{ }_{2} F_{1}\left[\begin{array}{c}
1, \frac{d-2}{2} ; \frac{p_{12}-p_{13}}{d-2} \\
m^{2}
\end{array}\right] \\
+\frac{1}{m^{2}} I_{2}^{(d)}\left(0, m^{2} ; 0\right) F_{1}\left(1,1,2-\frac{d}{2}, \frac{d}{2} ; \frac{p_{12}-p_{13}}{m^{2}}, \frac{p_{12}}{m^{2}}\right)
\end{gathered}
$$

where $F_{1}$ is the Appell hypergeometric function [10] which admits a simple one-fold integral representation:

$$
F_{1}\left(1,1,2-\frac{d}{2}, \frac{d}{2} ; x, y\right)=\frac{(d-2)}{2} \int_{0}^{1} d u \frac{[(1-u)(1-y u)]^{\frac{d}{2}-2}}{(1-x u)}
$$

Thus by using (3.38) one can obtain the result for the integral $I_{3}^{(d)}$ in terms of the Appell function $F_{1}$ and Gauss's hypergeometric function ${ }_{2} F_{1}$. This result is in agreement with the result obtained in Ref. [11] and later in Ref. [12]. At $d=4$ the result for $I_{3}^{(4)}$ in terms of Appell function $F_{3}$ was obtained in Ref. [13].

The Appell function $F_{1}$ in formula (3.38) has branch points if

$$
\left|p_{12}\right| \geq m^{2}, \quad \text { or } \quad\left|p_{12}-p_{13}\right| \geq m^{2} \text {. }
$$

To analytically continue the integral $I_{3}^{(d)}\left(0, m^{2}, 0 ; 0, p_{13}, p_{12}\right)$ into regions (3.40) one can use appropriate functional equations. When $\left|p_{12}\right| \geq m^{2}$, the following functional equation can be applied:

$$
\begin{gathered}
I_{3}^{(d)}\left(0, m^{2}, 0 ; 0, p_{13}, p_{12}\right)=\frac{m^{2}}{p_{12}} I_{3}^{(d)}\left(0, m^{2}, 0 ; 0, \frac{m^{2}\left(p_{13}-p_{12}+m^{2}\right)}{p_{12}}, \frac{m^{4}}{p_{12}}\right) \\
+\frac{\left(p_{12}-m^{2}\right)}{p_{12}} I_{3}^{(d)}\left(0,0,0 ; \frac{m^{2}\left(p_{13}-p_{12}+m^{2}\right)}{p_{12}}, \frac{\left(p_{12}-m^{2}\right)^{2}}{p_{12}}, p_{13}\right) .
\end{gathered}
$$


This relation can be derived from Eq. (3.26) with $m_{1}^{2}=m_{3}^{2}=m_{4}^{2}=p_{23}=0$ and $m_{2}^{2}=m^{2}$ by imposing the following conditions:

$$
G_{4}=0, \quad \partial_{1} \Delta_{4}=0, \quad \partial_{1} \partial_{3} \Delta_{4}=0 .
$$

On the right - hand side of the relation (3.41) the last two arguments of the integral $I_{3}^{(d)}$ in the first term are finite for large $\left|p_{12}\right|$.

If $\left|p_{12}-p_{13}\right| \geq m^{2}$ and $\left|p_{12}\right| \leq m^{2}$, the following relation can be applied

$$
\begin{aligned}
I_{3}^{(d)}(0, & \left.m^{2}, 0 ; 0, p_{13}, p_{12}\right)=\frac{p_{12} m^{2}}{m^{2} p_{13}+p_{12} p_{13}-p_{12}^{2}} I_{3}^{(d)}\left(0, m^{2}, 0 ; 0, \frac{p_{12}^{2}\left(p_{13}-p_{12}+m^{2}\right)}{m^{2} p_{13}+p_{12} p_{13}-p_{12}^{2}}, p_{12}\right) \\
& +\frac{p_{12}\left(p_{13}-p_{12}\right)}{m^{2} p_{13}+p_{12} p_{13}-p_{12}^{2}} I_{3}^{(d)}\left(0,0,0 ; \frac{m^{2}\left(p_{13}-p_{12}\right)^{2}}{m^{2} p_{13}+p_{12} p_{13}-p_{12}^{2}}, \frac{p_{12}^{2}\left(p_{13}-p_{12}+m^{2}\right)}{m^{2} p_{13}+p_{12} p_{13}-p_{12}^{2}}, p_{13}\right) \\
& +\frac{m^{2}\left(p_{13}-p_{12}\right)}{m^{2} p_{13}+p_{12} p_{13}-p_{12}^{2}} I_{3}^{(d)}\left(0, m^{2}, 0 ; 0, \frac{m^{2}\left(p_{13}-p_{12}\right)^{2}}{m^{2} p_{13}+p_{12} p_{13}-p_{12}^{2}}, 0\right) .
\end{aligned}
$$

This relation can be derived from Eq. (3.26) with $m_{1}^{2}=m_{3}^{2}=m_{4}^{2}=p_{23}=p_{24}=0$ and $m_{2}^{2}=m^{2}$ by imposing the following conditions:

$$
G_{4}=0, \quad \partial_{1} \Delta_{4}=0 .
$$

The penultimate argument of the first integral on the right hand side of (3.43) is finite for large values of $\left|p_{13}\right| \geq m^{2}$ if $p_{12} \neq-m^{2}$. The second and the third integrals on the right - hand side of this relation can be expressed in terms of the hypergeometric function ${ }_{2} F_{1}$ and their analytic continuation causes no problems.

If both conditions (3.40) hold then the analytic continuation can be done by applying both (3.41) and (3.43).

\section{Conclusions}

Finally, we summarize what we have accomplished in this paper.

First of all, we formulated the general method for deriving functional equations for Feynman integrals.

Second, it was shown that integrals with many kinematic arguments can be reduced to a combination of integrals with simpler kinematics.

Third, we demonstrated that our functional equations can be used for the analytic continuation of Feynman integrals to all kinematic domains.

In the present paper we considered rather particular cases of functional equations. The systematic investigation and classification of the proposed functional equations requires application of the methods of algebraic geometry and group theory.

A detailed consideration of our functional equations and their application to the one-loop integrals with four, five and six external legs as well as to some two- and three- loop Feynman integrals will be presented in future publications.

\section{Acknowledgment}

I am very thankful to Ronald Reid-Edwards for carefully reading the manuscript and useful remarks. This work was supported in part from DFG grants DFG KN365/3 and BMBF 
05HT6GUA. Part of this investigation was done during my stay at the Institut für Theoretische Physik E, RWTH Aachen where I was supported from the DFG grant Sonderforschungsbereich Transregio 9-03.

\section{References}

[1] B. Petersson, J. Math. Phys. 6, (1965) 1955.

[2] G. 't Hooft and M. J. G. Veltman, Nucl. Phys. B 44, (1972) 189.

[3] F. V. Tkachov, Phys. Lett. B 100 (1981) 65;

K. G. Chetyrkin and F. V. Tkachov, Nucl. Phys. B 192 (1981) 159.

[4] O. V. Tarasov, Phys. Rev. D 54 (1996) 6479 arXiv:hep-th/9606018.

[5] J. Fleischer, F. Jegerlehner and O. V. Tarasov, Nucl. Phys. B 566 (2000) 423 arXiv:hep-ph/9907327.

[6] C. G. Bollini and J. J. Giambiagi, Phys. Lett. B 40 (1972) 566.

[7] E. E. Boos and A. I. Davydychev, Theor. Math. Phys. 89, (1991) 1052; Teor. Mat. Fiz. 89, (1991) 56 .

[8] A. Erdèly et. al., Higher Transcendental Functions Vol.1, McGraw-Hill, New York, 1953.

[9] A. I. Davydychev, Phys. Rev. D 61 (2000) 087701 arXiv:hep-ph/9910224.

[10] P. Appell and J. Kampé de Fériet Fonctions hypergeometriques et hyperspériques, Gauthier Villars, Paris, 1926.

[11] J. Fleischer, F. Jegerlehner and O. V. Tarasov, Nucl. Phys. B 672, (2003) 303, arXiv:hep-ph/0307113.

[12] A. I. Davydychev, Nucl. Instrum. Meth. A 559 (2006) 293 |arXiv:hep-th/0509233|.

[13] L. G. Cabral-Rosetti and M. A. Sanchis-Lozano, J. Comput. Appl. Math. 115 (2000) 93 arXiv:hep-ph/9809213. 